\title{
Dynamic CT angiography for therapy evaluation after transarterial chemoembolization of hepatocellular carcinoma
}

Acta Radiologica

2020, Vol. 6I(2) |48-155

(C) The Foundation Acta Radiologica 2019

Article reuse guidelines:

sagepub.com/journals-permissions DOI: $10.1|77 / 0284| 8511985460$ journals.sagepub.com/home/acr

(SAGE

\author{
Marc Saake (D), Hannes Seuss, Matthias Hammon, \\ Stephan Ellmann (D, Matthias May, Michael Uder and \\ Axel Schmid
}

\begin{abstract}
Background: Liver dynamic computed tomography (CT) is an established method for pre- and post-interventional evaluation of hepatocellular carcinoma. To date only the liver parenchyma and perfusion information of dynamic CT has been evaluated widely.

Purpose: To evaluate the vascular information contained in dynamic CT datasets.

Material and Methods: Dynamic CT performed one day after transarterial chemoembolization $(60 \mathrm{~mL}$ of contrast medium, $6 \mathrm{~mL} / \mathrm{s}, 40 \mathrm{~s}$ scan duration) were retrospectively evaluated. Conventional slice and angiographic maximumintensity-projection reconstructions were calculated on a multi-modality post-processing platform. Datasets were evaluated for viable tumor, anatomy of the vasculature, and potential tumor-feeding vessels. The results were compared to digital subtraction angiography images.

Results: In total, 94 treated hepatocellular carcinoma nodules were evaluated (62 dynamic CT scans, 46 patients $[34$ men; mean age $=69$ years]). Forty-six partially viable tumors were diagnosed after transarterial chemoembolization. In all of these, tumor-feeding vessels were found in dynamic CT. Seventeen suspected extra-hepatic tumor feeders were reported, of which 14 had not been found during previous transarterial chemoembolization.

Conclusion: Dynamic CT is useful in post-interventional imaging of hepatocellular carcinoma after transarterial chemoembolization due to its ability to detect residual viable tumor parts and to show previously unknown intra- and extrahepatic tumor-feeding vessels.
\end{abstract}

\section{Keywords}

Abdomen, computed tomography angiography, interventional, chemoembolization, liver

Date received: II February 2019; accepted: 10 May 2019

\section{Introduction}

Hepatocellular carcinoma (HCC) is the sixth most common cancer and the third most common cause of death from cancer worldwide (1). Transarterial chemoembolization (TACE) is the most widely used primary loco-regional therapy for unresectable HCC and the recommended first-line therapy for patients in the intermediate stage of the disease (2). However, tumor response to TACE can be variable (3). Digital subtraction angiography (DSA) and fluoroscopy are the standard imaging techniques to visualize the hepatic vasculature and to identify tumor-feeding arteries during TACE, while cone beam computed tomography (CBCT) has become increasingly important for treatment planning in chemoembolization and other

Department of Radiology, University of Erlangen-Nuremberg, Germany

\section{Corresponding author:}

Marc Saake, Department of Radiology, University Hospital Erlangen, Maximiliansplatz 3, 91054 Erlangen, Germany.

Email: Marc.Saake@uk-erlangen.de 
embolotherapies. However, CBCT is implemented only in high-end C-arm fluoroscopy units and is therefore not available in all interventional liver procedures. In many institutions, computed tomography (CT) or magnetic resonance imaging (MRI) is performed shortly after TACE to evaluate therapy outcome. Further TACE treatment is usually scheduled in patients with residual viable tumor in imaging (4).

Liver dynamic CT (dCT) can be used for postinterventional imaging after TACE. In this scan mode, multiple repetitive scans of the liver are performed while a contrast bolus flows through the vessels and liver parenchyma $(5,6)$. This four-dimensional (4D) dataset can be reconstructed in different ways: conventional slice reconstructions demonstrate possible contrast-enhancing tumor remnants. Color-coded liver perfusion maps visualize liver tissue and tumor perfusion (7). The value of these parameter reconstructions for the detection and characterization of HCCs is already known $(8,9)$.

However, there are little reports on the value of the vascular information contained in $\mathrm{dCT}$ datasets. Thinslice reconstructions of the $\mathrm{dCT}$ raw data with secondary multi-planar reconstructions (MPR) and maximum intensity projections (MIP) allow high-resolution evaluation of the vasculature, comparable to conventional CT angiography (CTA). In contrast to CTA, optimal arterial phase contrast between tumor feeding arteries and surrounding tissue can be consistently achieved with $\mathrm{dCT}$, since it provides imaging data from multiple consecutive points in time. Moreover, dCT offers visualization of both intrahepatic and extrahepatic arteries and therefore allows the identification of extrahepatic tumor feeding vessels without the need of selective catheterization as in DSA or CBCT imaging. Therefore, angiographic reconstructions of $\mathrm{dCT}$ data might be helpful in case of residual tumors, particularly if additional extra-hepatic tumor feeders could not be identified during previous TACE procedure, which might be the case especially if CBCT imaging was not available during the TACE procedure.

In summary, the aim of this study was to evaluate the usefulness of $\mathrm{dCT}$ for liver imaging after TACE, with particular focus on the value of angiographic reconstructions.

\section{Methods}

\section{Study population}

Institutional review board approval was obtained before the beginning of this retrospective analysis. Informed consent of the patients was obtained. All clinical procedures based on local tumor board decisions.
Study inclusion criteria were HCC in cirrhotic liver and the performance of $\mathrm{dCT}$ within three days after TACE in our department. A total of 53 consecutive patients met the inclusion criteria. Exclusion criteria were age $<18$ years and incomplete data. Seven patients had to be excluded from the analysis due to missing thin slice datasets.

In total, 46 patients $(34$ men; mean age $=69$ years; age range $=49-86$ years) were included in the analysis. In these, 62 TACE procedures with consecutive dCTs (22 initial TACEs, 40 TACEs after previous TACE or radiofrequency ablation and $\mathrm{HCC}$ recurrence at local or distant site) had been performed. Mean time between TACE and dCT was one day (range $=0-2$ days).

Diagnosis of HCC based on histology or imaging criteria according to the 2011 guidelines on HCC by the American Association for the Study of Liver Diseases (AASLD) (10). Liver MRI was performed before the intervention in all patients and after the intervention in 59/62 TACE procedures. In three procedures, there was no post-interventional MRI. If residual viable tumor was present in post-TACE dCT or MRI, a TACE repetition was performed in all patients.

\section{Transarterial chemoembolization (TACE)}

TACE was performed using DSA with epirubicin loaded drug-eluting beads (DC Bead; Biocompatibles UK Ltd., Surrey, UK; bead size $=100-300 \mu \mathrm{m}$; maximum dose of epirubicin $=50 \mathrm{mg}$ ) delivered from superselective microcatheter positions under fluoroscopy until hemostasis was reached. If the amount of epirubicin loaded beads was not sufficient to reach hemostasis, the procedure was continued using bland embolization (Bead block; Biocompatibles UK Ltd., Surrey, UK; up to a maximum of two additional vials of $100-300 \mu \mathrm{m}$ beads). At the end of each TACE, a proper hepatic artery DSA was performed. Cone-beam CT was not available at our institution during the study period.

\section{Dynamic CT of the liver (dCT)}

According to local institutional guidelines, performance control after TACE was done by dCT. All dCTs were performed on a 128-slice CT scanner (Somatom Definition AS+; Siemens, Erlangen, Germany) using a 4D spiral protocol with $20.7-\mathrm{cm}$ coverage in z-axis. Ten consecutive scans in cranio-caudal direction were performed within $40 \mathrm{~s}$, with each scan and the subsequent table reposition to the start position lasting $2 \mathrm{~s}$, respectively. All patients received special breathing training before dCT. Only patients 
capable of holding their breath for $40 \mathrm{~s}$ were examined by dCT.

Aortic bolus arrival time was measured by test bolus with an injection of $5 \mathrm{~mL}$ contrast medium (CM). dCT was performed during breath-hold in inspiration with a scan start time of $8 \mathrm{~s}$ before the measured aortic testbolus peak. A total of $60 \mathrm{~mL}$ of iodinated CM (iomeprol $350 \mathrm{mg}$ iodine $/ \mathrm{mL}$, Imeron 350; Bracco Imaging, Konstanz, Germany), followed by $30 \mathrm{~mL}$ of saline flush, were injected via an 18-gauge cannula into a cubital vein at a rate of $6 \mathrm{~mL} / \mathrm{s}$ using a doublepiston power injector (Accutron CT2; Medtron, Saarbruecken, Germany).

$\mathrm{dCT}$ parameters were: tube voltage $=100 \mathrm{kV}$; tube current-time product $=150 \mathrm{mAs} ;$ collimation $=128 \times$ $0.6 \mathrm{~mm}$; rotation time $=0.3 \mathrm{~s} ; \quad \mathrm{CT}$ dose index volume $=71.02 \mathrm{mGy}$. The effective radiation dose of the complete dCT was calculated as $22.1 \mathrm{mSv}$, based on the dose-length product and the conversion factor of $0.015 \mathrm{mSv} \times \mathrm{mGy}^{-1} \times \mathrm{cm}^{-1}$ (11).

A primary thin-slice reconstruction was performed: section thickness $=1.5 \mathrm{~mm}$; increment $=1 \mathrm{~mm}$; soft reconstruction kernel $=\mathrm{B} 20 \mathrm{f}$; number of images $=10 \times$ $206=2060$.

\section{Image processing}

Image evaluation was performed using a clinical multimodality post-processing platform (syngo.plaza/syngo. via; Siemens, Erlangen, Germany). dCT datasets were analyzed in a dedicated 4D software tool (syngo.via VA30, CT Body Perfusion; Siemens, Erlangen, Germany), consisting of a 3D tool with real-time MPR and MIP capability and the additional possibility to quickly switch between the 10 points in time of each dCT. Liver parenchyma was evaluated using $5-\mathrm{mm}$ thick MPRs. Vessels were evaluated in MPR and MIP mode with variable slice thickness at the readers' choice. DSA images were presented in a standard PACS software offering subtracted and unsubtracted display mode (syngo.plaza VB20A; Siemens, Erlangen, Germany).

\section{Image evaluation}

Review and analysis of the images was performed in consensus reading by two board-certified radiologists with 20 and 10 years of experience in abdominal imaging and interventional radiology, respectively. Readers had access to the pre-interventional images (CT and/or MRI), the TACE DSA images, and the postinterventional MR images.

For all dCT examinations, image quality $(0=$ non-diagnostic, $1=$ diagnostic with limitations, $2=$ diagnostic, $3=$ good, $4=$ excellent) and presence of motion artefacts $(0=$ none, $1=$ mild, $2=$ severe $)$ were scored by the readers. Anatomy of the hepatic vasculature was classified using the Michels' scheme (12). This classification system describes frequent anatomical variations of the hepatic arterial system (Table 1).

The datasets were evaluated for contrast-enhancing tumor remnants using the slice images first. Residual nodal arterial contrast uptake in the region of the treated tumor was regarded as HCC remnant. Focal hyperdensity in the native phase of $\mathrm{dCT}$ without further density increase in consecutive phases was classified as contrast deposit after TACE. In patients with multifocal $\mathrm{HCC}$, only tumors treated during the prior TACE were evaluated. The points in time of subjective optimal vessel contrast for each DCT and optimal tumor-to-liver-contrast for each tumor remnant were determined by visually comparing the different dCT contrast phases with the $4 \mathrm{D}$ software tool. In case of viable tumor remnants, for each tumor the size of the viable remnant was measured according to mRECIST (13). Potential tumor-feeding vessels were identified and the likelihood of tumor supply was estimated for each vessel using a 5 -point score $(0=$ vessel far distant to tumor, tumor supply very unlikely, $1=$ vessel distant to tumor, tumor supply unlikely, $2=$ vessel in tumor proximity, tumor supply possible, $3=$ vessel close to tumor, tumor supply likely, $4=$ vessel traceable directly to tumor, tumor supply very likely). Furthermore, each tumor-feeding vessel was analyzed regarding its fulllength coverage in the acquired image material. Finally, the readers reported the following auxiliary findings when present: extra-hepatic metastases; other

Table I. Michel's classification and reported hepatic arterial supply in our cohort.

\begin{tabular}{lll}
\hline Michel's type & Description & $\mathrm{n}$ \\
\hline 1 & Standard anatomy & 48 \\
2 & Replaced LHA from LGA & 4 \\
3 & Replaced RHA from SMA & 2 \\
4 & Replaced RHA and LHA & 2 \\
5 & Accessory LHA from LGA & 4 \\
6 & Accessory RHA from SMA & 2 \\
7 & Accessory LHA and RHA & 0 \\
8 & Replaced LHA + accessory & 0 \\
& RHA or replaced & \\
9 & RHA + accessory LHA & \\
10 & CHA from SMA & 0 \\
\hline
\end{tabular}

Variant hepatic arterial supply was found in 14 dCTs.

CHA: common hepatic artery; LGA: left gastric artery; LHA: left hepatic artery; PHA: proper hepatic artery; RHA: right hepatic artery; SMA: superior mesenteric artery. 
tumors; thrombosis of portal vein; ascites; and other potentially relevant findings.

Post-interventional MR images were evaluated for viable tumor remnants in an independent second read.

\section{Clinical procedure}

To study the clinical importance of post-interventional $\mathrm{dCT}$, the readers assessed whether each $\mathrm{dCT}$ was suitable to evaluate the TACE outcome. In case of residual viable tumor, the readers noted whether the tumor remnants had already been present in the final TACE images and if residual tumor-feeding vessels were previously identified by DSA imaging. Moreover, they judged if TACE repetition was likely to be successful and if $\mathrm{dCT}$ was sufficient for planning of TACE repetition.

\section{Intra- and extra-hepatic feeders}

A second reading session was performed by the same readers to further study the potential intra- and extrahepatic tumor-feeding vessels reported in dCT. All available DSA images from prior and subsequent examinations of these patients were evaluated with regard to whether the suspected tumor-feeding vessels had been catheterized and if they supplied the tumors.

\section{Data analysis}

Data analysis was performed using Microsoft Excel 2010 (Microsoft Corporation, Redmond, WA, USA). Quantitative values are given as mean \pm standard deviation.

\section{Results}

\section{Image evaluation}

All examinations were evaluable. Image quality was good or better in all dCT scans (excellent $=36$, good $=26$ ). Motion artefacts were present in none of the examinations. Variant hepatic arterial supply was found in $14 \mathrm{dCT}$ (Table 1). Mean point in time of optimal vessel contrast was $16 \mathrm{~s}$ after scan start (range $=4-24 \mathrm{~s}$ ).

In total, 94 HCC nodules treated by TACE were evaluated. After TACE, 46 partially viable nodules were found in $34 \mathrm{dCTs}$. In 14 of these 46 nodules, contrast deposits from prior TACE were present (directly adjacent to the viable tumor remnant $=1$, in the same nodule but distant to the viable tumor part $=13$ ).

Mean point in time of optimal tumor-to-livercontrast was $20 \mathrm{~s}$ after scan start (range $=8-36 \mathrm{~s}$ ). Mean post-TACE viable tumor size was $2.7 \pm 1.3 \mathrm{~cm}$.
In 32 post-interventional MR scans 44 viable tumor nodules were reported. The missing two tumor nodules compared to the post-interventional dCTs were reported in patients without post-interventional MRI.

Potential tumor-feeding vessels could be identified for each residual viable $\mathrm{HCC}$ in $\mathrm{dCT} ; 71$ potential tumor-feeding vessels were reported: 54 (76\%) segmental branches of the proper hepatic artery and the cystic artery; and $17(24 \%)$ vessels with an extra-hepatic origin. There was one vessel in 28 tumors, two vessels in 11 tumors, and three vessels in seven tumors. The likelihood of tumor supply was estimated "tumor supply very likely" for 31 arteries, "tumor supply likely" for 30 arteries, "tumor supply possible" for eight arteries, and "tumor supply unlikely" for two arteries. In all arteries but one (superior epigastric artery), the feeding vessels were completely depicted within the scan range. Table 2 lists the reported potential tumorfeeding vessels.

\section{Clinical impact}

All dCTs were reported as suitable to evaluate the TACE outcome. In 22 of $34 \mathrm{dCTs}$ with residual viable tumors, the dCT showed viable tumors which were not suspected in the corresponding DSA images. In 12 of $34 \mathrm{dCT}$ s with residual viable tumors, the tumor remnants had been previously known from the DSA images. However, complete embolization could not be achieved in these procedures due to the large size of tumors and complete consumption of the embolization material. In 32 of 34 cases, TACE repetition was regarded technically feasible and all dCTs were reported as sufficient for planning of TACE repetition. In two of these 34 , the readers discouraged from TACE repetition (HCC supply from the right colic artery with risk of colon necrosis $=1$, large TACE-induced liver necrosis with uncertain remaining tumor feeders $=1$ ).

Table 2. Reported potential tumor-feeding vessels in 46 residual viable HCCs.

\begin{tabular}{ll}
\hline Potential tumor-feeding vessel & $\mathrm{n}$ \\
\hline Intra-hepatic & \\
Hepatic segment arteries & 48 \\
Cystic artery & 6 \\
Extra-hepatic & 8 \\
Right phrenic artery & 3 \\
Left phrenic artery & 2 \\
Left gastric artery & $\mathrm{I}$ \\
Right gastric artery & $\mathrm{I}$ \\
Right gastroepiploic artery & $\mathrm{I}$ \\
Right colic artery & $\mathrm{I}$ \\
Left superior epigastric artery & $\mathrm{In}$
\end{tabular}

Of the suspected tumor feeders, $24 \%$ were of extra-hepatic origin. In some tumors, more than one suspected feeding vessels were reported. 
Radiofrequency ablation was reported as a possible treatment alternative in $14 \mathrm{dCTs}$ and was recommended as the best therapeutic option in $10 \mathrm{dCTs}$. Extra-hepatic findings which would have an impact on the further procedure in a clinical setting were reported in two dCTs (gallbladder perforation $=1$, previously unknown adrenal metastasis $=1$ ). Overall, clinical impact was reported in 24 of $62 \mathrm{dCTs}(39 \%)$ due to previously unknown residual tumor and extrahepatic findings.

\section{Intra- and extra-hepatic feeders}

In $\mathrm{dCT}, 54$ potential intra-hepatic tumor-feeding vessels were reported. Of these, 48 had been studied by DSA. Of these, $45(94 \%)$ turned out to be actually tumor-feeding. All three false-positive vessels had a low likelihood of tumor supply score ("unlikely" or "possible"). Four of the reported intra-hepatic feeders had not been catheterized.

Furthermore, 17 suspected extra-hepatic tumor feeders were described in dCT, 14 of which had not been diagnosed during previous TACE (Figs. 1-3). 11 of the suspected 17 vessels had been catheterized, confirming eight $(73 \%)$ as actually tumor-feeding. All of the three false-positive vessels had a low likelihood of tumor supply score ("unlikely" or "possible"). Six of the suspected 17 extra-hepatic tumor feeders found in this retrospective analysis had been missed in the initial clinical image reading and had not been catheterized.

\section{Discussion}

The aim of this study was to evaluate the usefulness of $\mathrm{dCT}$ for liver imaging after TACE in patients with
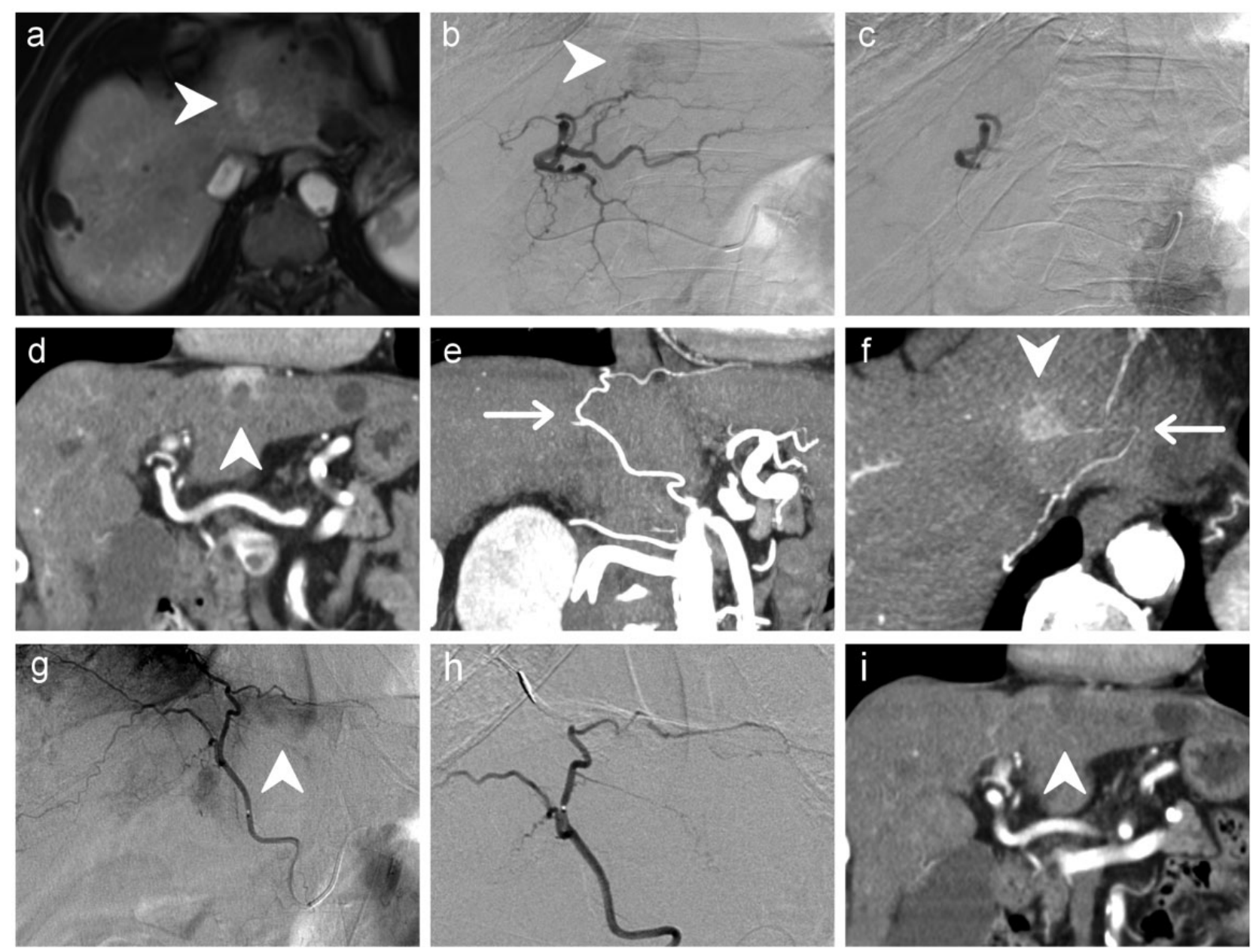

Fig. I. A 75-year-old man with HCC in left liver lobe. (a) Transversal, TI-weighted, arterial phase MRI shows HCC in left liver lobe (arrowhead). (b) DSA demonstrates tumor supply from left liver artery (arrowhead). (c) TACE was performed until hemostasis was reached. (d) Coronal MPR of post-TACE dCT shows viable residual tumor (arrowhead). (e) Coronal and (f) transversal MIP of dCT demonstrate branch of right phrenic artery traceable directly to tumor (arrow, likelihood score 4). (g) Subsequent DSA confirms HCC supply from right phrenic artery. (h) After protective coil embolization of a phrenic branch, TACE of the right phrenic artery was performed. (i) Coronal MPR of follow-up dCT shows avascular tumor (arrowhead). 

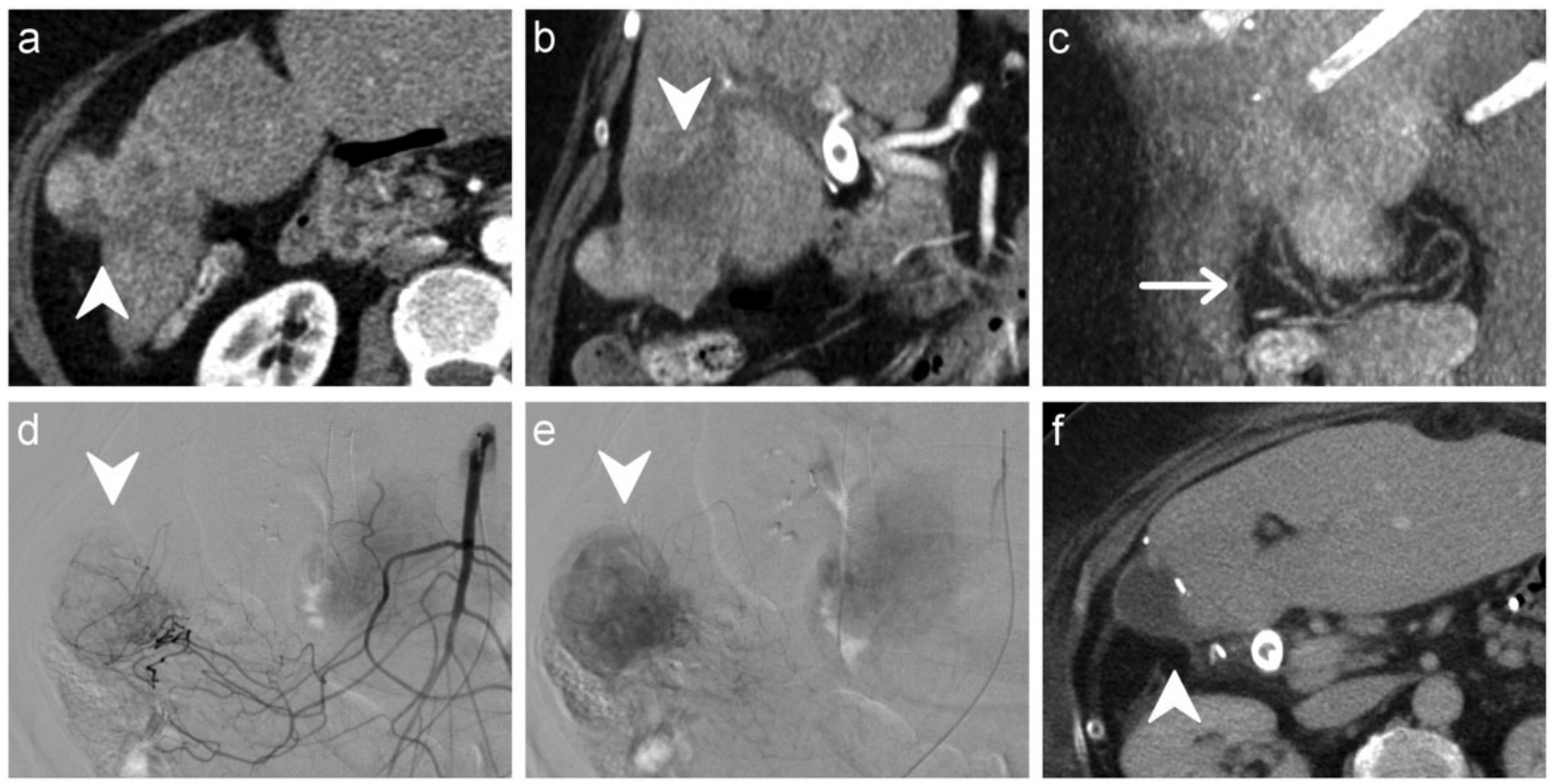

Fig. 2. A 69-year-old woman with HCC in right liver lobe, transgressing the liver capsule. (a) Transversal and (b) coronal MPR of post-TACE dCT show viable residual tumor (arrowheads). (c) Sagittal MIP of dCT demonstrates branches of right colic artery traceable directly to tumor (arrow, likelihood score 4). (d, e) DSA confirms tumor supply from right colic artery (arrowheads point to tumor). (f) Follow-up CT after liver segment resection and right hemicolectomy shows bilioma at resection margin (arrowhead) without evidence of viable tumor.
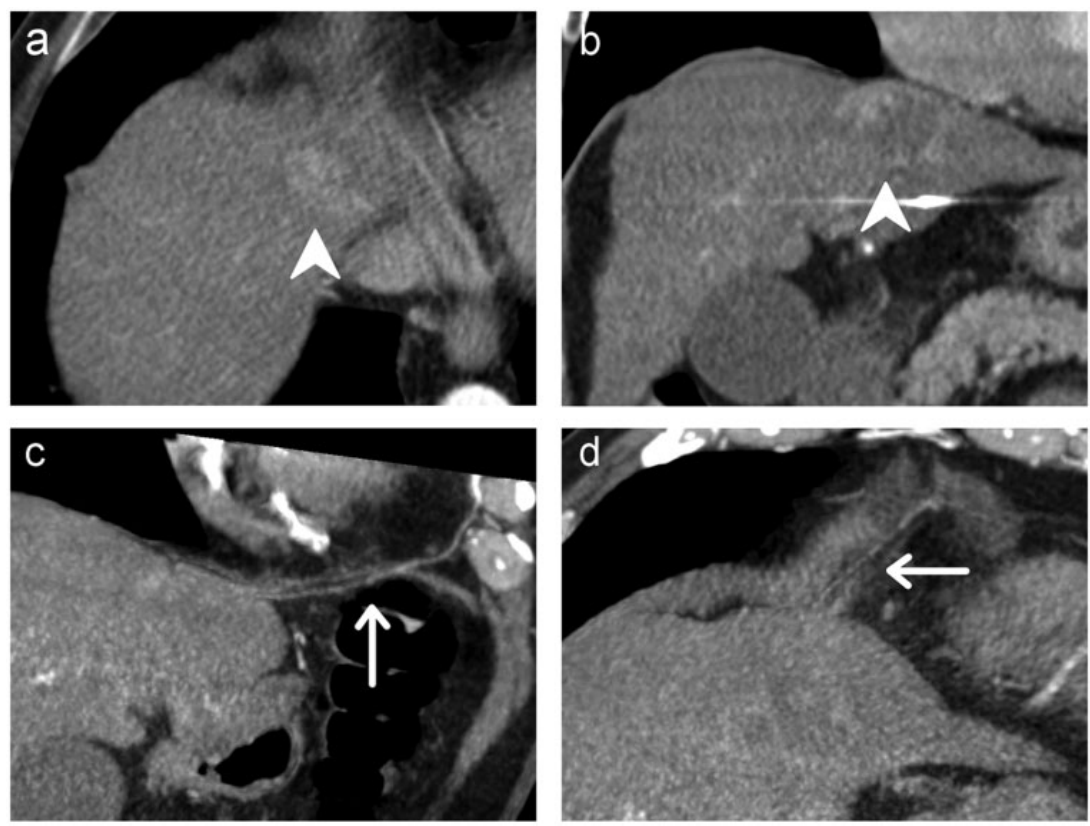

Fig. 3. A 67-year-old man with HCC in left liver lobe. (a) Transversal and (b) coronal MPR of post-TACE dCT show viable residual tumor (arrowheads). (c) Oblique and (d) transversal MIPs of dCT demonstrate branches of left superior epigastric artery traceable close to tumor (arrows, likelihood score 3). DSA confirmation of tumor supply was not available in this patient.

HCC. We found that dCT can demonstrate residual viable tumor parts and potential tumor-feeding vessels. To our knowledge, this is the first paper on the additional value of angiographic information of $\mathrm{dCT}$.
The goal of TACE in HCC is the induction of complete tumor necrosis. However, incomplete necrosis is frequent after TACE (14) and has been reported to be a risk factor for tumor recurrence (7). Therefore, 
follow-up imaging after TACE is essential for prompt detection of residual viable tumor parts, as these may require further therapy. Multiphase contrast-enhanced $\mathrm{CT}$ or MRI is the current standard for imaging of HCC (7).

The majority of HCCs feature hypervascularity in the arterial phase $(3,15)$. However, the point in time of optimal tumor-to-liver-contrast can vary (16). Thus, performing only a single arterial phase scan bears the potential to miss contrast-enhancing tumor parts. In our cohort, the points in time of optimal vessel and tumor-to-liver contrast differed largely between patients. However, due to the performance of multiple scans in dCT, an arterial phase scan with individually best contrast was available in all patients. Furthermore, the availability of both native and contrast-enhanced phases in $\mathrm{dCT}$ facilitated the identification of residual contrast-enhancing tumors and the differentiation from $\mathrm{CM}$ deposition from previous TACE. In case of residual viable tumor parts, the identification of tumor-feeding vessels can be helpful for planning of TACE repetition, as extra-hepatic HCC feeders can be difficult to detect during DSA. The performance of $\mathrm{dCT}$ after TACE might aid the detection of additional tumor-feeding vessels as non-occluded feeders may tend to dilate after embolization of main tumor feeders.

Anatomic variations in hepatic arterial blood supply are frequent (12). We found a variant arterial anatomy in $23 \%$ of patients. Of the suspected remnant tumor feeders, $24 \%$ were of extra-hepatic origin. Besides frequent extra-hepatic feeders like the phrenic arteries, we could identify unusual supplies like the left superior epigastric artery, which would not be catheterized during TACE without prior knowledge.

CBCT offers the visualization of HCCs and the supplying hepatic vasculature in high resolution and the intra-procedural feasibility of this technique is advantageous. There are reports that the use of CBCT can reduce the local tumor recurrence rate (17). However, contrast injection in CBCT is performed selectively via an intra-arterial catheter, typically into the common hepatic artery. This bears the risk to miss relevant extra-hepatic tumor supplies, not originating from the common hepatic artery. Here, dCT might be superior, as all arteries are contrasted simultaneously. Moreover, the world-wide availability of CBCT is still limited. In hospitals where CBCT is not available, dCT can assist interventional radiologists in detection of HCC feeders.

MRI is a proper alternative to detect residual viable tumors without use of ionizing radiation. In this study, all residual tumors were reported in both $\mathrm{dCT}$ and MRI, in patients where both modalities had been performed. However, we consider the additional angiographic information from dCT very helpful for planning of TACE repetition. We therefore propose the following clinical pathway: MRI could be performed after TACE as control of success in all patients. If viable HCC remnants are present, $\mathrm{dCT}$ could be used before TACE repetition, especially in case of unclear tumor supply.

To evaluate the clinical significance of $\mathrm{dCT}$ in the post-interventional setting, we simulated a clinical decision finding process: $39 \%$ of dCTs would have had significant influence on the further therapeutic decisions due to detection of tumor residues, previously unknown extra-hepatic tumor supply, and extrahepatic findings.

Our study has some limitations. First, it is of retrospective character. Thus, some potentially tumorfeeding vessels identified retrospectively in $\mathrm{dCT}$ at the study image read had not been catheterized and it remains unknown whether they were actually tumorfeeding or not. However, all vessels with a high dCT likelihood of tumor supply score which had been catheterized proved actually tumor-feeding. Second, we cannot compare $\mathrm{dCT}$ to $\mathrm{CBCT}$ as this technique was not available at our institution during the study period. Third, the retrospective data of our study does not include conventional $\mathrm{CT}$ images in the majority of the cases and therefore, $\mathrm{dCT}$ could not be compared to conventional CT in our cohort. Finally, the main focus of our study was the evaluation of the feasibility of angiographic reconstructions from $\mathrm{dCT}$ and their value for TACE planning. A future prospective study should evaluate, whether TACE planning using dCT has the potential to shorten the duration of the TACE procedure, to increase the ratio of complete tumor necrosis, and to improve clinical outcomes in follow-up.

In conclusion, $\mathrm{dCT}$ is useful in post-interventional imaging of HCC after TACE due to the valuable angiographic information on intra- and extra-hepatic tumor-feeding vessels. Additionally, dCT can demonstrate residual viable tumors.

\section{Declaration of conflicting interests}

The author(s) declared no potential conflicts of interest with respect to the research, authorship, and/or publication of this article.

\section{Funding}

The author(s) received no financial support for the research, authorship, and/or publication of this article.

\section{ORCID iDs}

Marc Saake (D) https://orcid.org/0000-0003-3520-0312

Stephan Ellmann (D) https://orcid.org/0000-0003-2737-5526 


\section{References}

1. Ferlay J, Shin HR, Bray F, et al. Estimates of worldwide burden of cancer in 2008: GLOBOCAN 2008. Int $\mathbf{J}$ Cancer 2010;127:2893-2917.

2. European Association for the Study of the Liver; European Organisation for Research and Treatment of Cancer. EASL-EORTC clinical practice guidelines: management of hepatocellular carcinoma. J Hepatol 2012;56:908-943.

3. Alba E, Valls C, Dominguez J, et al. Transcatheter arterial chemoembolization in patients with hepatocellular carcinoma on the waiting list for orthotopic liver transplantation. AJR Am J Roentgenol 2008;190:1341-1348.

4. Lencioni R, Petruzzi P, Crocetti L. Chemoembolization of hepatocellular carcinoma. Semin Intervent Radiol 2013;30:3-11.

5. Yanaga Y, Awai K, Nakaura T, et al. Optimal contrast dose for depiction of hypervascular hepatocellular carcinoma at dynamic CT using 64-MDCT. AJR Am J Roentgenol 2008;190:1003-1009.

6. Wu D, Tan M, Zhou M, et al. Liver computed tomographic perfusion in the assessment of microvascular invasion in patients with small hepatocellular carcinoma. Invest Radiol 2015;50:188-194.

7. Hennedige T, Venkatesh SK. Imaging of hepatocellular carcinoma: diagnosis, staging and treatment monitoring. Cancer Imaging 2013;12:530-547.

8. Fischer MA, Kartalis N, Grigoriadis A, et al. Perfusion computed tomography for detection of hepatocellular carcinoma in patients with liver cirrhosis. Eur Radiol 2015;25:3123-3132.

9. Hayano K, Desai GS, Kambadakone AR, et al. Quantitative characterization of hepatocellular carcinoma and metastatic liver tumor by CT perfusion. Cancer Imaging 2013;13:512-519.

10. Bruix J, Sherman M; American Association for the Study of Liver Diseases. Management of hepatocellular carcinoma: an update. Hepatology 2011;53:1020-1022.

11. AAPM Task Group 23. The measurement, reporting, and management of radiation dose in CT. AAPM Report No. 96. College Park, MD: 2007.

12. Michels NA. Newer anatomy of the liver and its variant blood supply and collateral circulation. Am J Surg 1966;112:337-347.

13. Lencioni R, Llovet JM. Modified RECIST (mRECIST) assessment for hepatocellular carcinoma. Semin Liver Dis 2010;30:52-60.

14. Huang J, He X, Lin X, et al. Effect of preoperative transcatheter arterial chemoembolization on tumor cell activity in hepatocellular carcinoma. Chin Med J (Engl) 2000;113:446-448.

15. Lee JH, Lee JM, Kim SJ, et al. Enhancement patterns of hepatocellular carcinomas on multiphasic multidetector row CT: comparison with pathological differentiation. Br J Radiol 2012;85:e573-583.

16. Kagawa Y, Okada M, Yagyu Y, et al. Optimal scan timing of hepatic arterial-phase imaging of hypervascular hepatocellular carcinoma determined by multiphasic fast CT imaging technique. Acta Radiol 2013;54:843-850.

17. Miyayama S, Yamashiro M, Hashimoto $M$, et al. Comparison of local control in transcatheter arterial chemoembolization of hepatocellular carcinoma $\leq 6 \mathrm{~cm}$ with or without intraprocedural monitoring of the embolized area using cone-beam computed tomography. Cardiovasc Intervent Radiol 2014;37:388-395. 